\title{
On diffeomorphisms deleting weak compacta in Banach spaces
}

\author{
by \\ Daniel Azagra and Alejandro Montesinos (Madrid)
}

\begin{abstract}
We prove that if $X$ is an infinite-dimensional Banach space with $C^{p}$ smooth partitions of unity then $X$ and $X \backslash K$ are $C^{p}$ diffeomorphic for every weakly compact set $K \subset X$.
\end{abstract}

1. Introduction, main results and preliminaries. In 1953 Victor L. Klee [20] proved that, if $X$ is a non-reflexive Banach space or an infinitedimensional $L^{p}$ space and $K$ is a compact subset of $X$, there exists a homeomorphism between $X$ and $X \backslash K$ which is the identity outside a given neighborhood of $K$. Klee also proved that for those infinite-dimensional Banach spaces $X$ the unit sphere and the unit ball are homeomorphic to any of the closed hyperplanes in $X$, and gave a topological classification of convex bodies in Hilbert spaces. In subsequent papers, Bessaga and Klee generalized those results to every infinite-dimensional normed space [10, 11, 12].

Klee's original proofs were of a strong geometrical flavor: very beautiful, but rather difficult to handle in an analytical way. Nevertheless, C. Bessaga found elegant explicit formulas for deleting homeomorphisms, based on the existence of continuous noncomplete (nonequivalent) norms in every infinitedimensional Banach space. This discovery allowed him in 1966 to construct diffeomorphisms which delete points in the Hilbert space, and to prove that the Hilbert space is diffeomorphic to its unit sphere [8]. These striking results have been highly celebrated and they remain a key ingredient in the proofs of the already classic fundamental theorems on Hilbert manifolds (e.g., that any two homotopic Hilbert manifolds are diffeomorphic, see [13, 17, 22]).

These kinds of results about topological negligibility have also found many interesting applications in several branches of mathematics, which include

2000 Mathematics Subject Classification: 46B20, 57R50, 58B99.

D. Azagra was supported by a Marie Curie Fellowship of the European Community Training and Mobility of Researchers Programme under contract number HPMF-CT2001-01175. A. Montesinos was supported by a FPI Fellowship from the Comunidad de Madrid. 
fixed point theory, smooth topological classification of convex bodies, strange phenomena concerning ordinary differential equations and dynamical systems in infinite dimensions, the failure of Rolle's theorem in infinite dimensions and many more things; see $[4,5,9,3,7]$ and the references therein. Very recently, Manuel Cepedello and the first-named author have used smooth topological negligibility to prove the following approximate strong version of the Morse-Sard theorem: the smooth functions with no critical points are dense in the space of continuous functions on every Hilbert manifold [2].

In view of the interest of such applications, it is natural to try to extend these results to Banach spaces other than the Hilbert space.

The real-analytic and smooth negligibility of compact sets in Banach spaces was first studied by Tadeusz Dobrowolski [15], who showed that for every infinite-dimensional Banach space $X$ having a $C^{p}$ noncomplete norm, and for every compact set $K$ in $X$, the space $X$ is $C^{p}$ diffeomorphic to $X \backslash K$. Unfortunately, it is still unknown whether every Banach space with a $C^{p}$ smooth equivalent norm has a noncomplete $C^{p}$ smooth norm as well. Nevertheless, without showing the existence of smooth noncomplete norms, it was recently proved in [4] that every Banach space $(X,\|\cdot\|)$ with a $C^{p}$ smooth norm $\varrho$ is $C^{p}$ diffeomorphic to $X \backslash K$.

Despite all these efforts, the natural question as to the characterization of those Banach spaces in which compact sets are topologically negligible remains open. This is due to a surprising (and rather uncomfortable) theorem proved by R. Haydon $[18,19]$ : there are Banach spaces which have $C^{\infty}$ smooth bump functions, and even $C^{\infty}$ smooth partitions of unity, but no equivalent $C^{1}$ smooth norm.

In this paper we deal with the following natural question: what can be said about smooth negligibility of compacta in those Banach spaces with smooth partitions of unity? As we have just pointed out, there are Banach spaces with smooth partitions of unity which have no equivalent smooth norms, and therefore the known results on diffeomorphisms deleting compacta are useless in this setting. Nevertheless, we will prove the following.

THEOREM 1.1. Let $X$ be an infinite-dimensional Banach space which has $C^{p}$ smooth partitions of unity, and $p \in \mathbb{N} \cup\{\infty\}$. Then, for every weakly compact set $K \subset X$ and every starlike body $A$ such that $\operatorname{dist}(K, X \backslash A)>0$, there exists a $C^{p}$ diffeomorphism $h: X \longrightarrow X \backslash K$ such that $h$ is the identity outside A.

In particular, when $K$ is compact and $K \subset \operatorname{int}(A)$, there always exists such a deleting diffeomorphism $h$.

The class of Banach spaces which admit smooth partitions of unity is quite large (see [14]). On the other hand, it is an open problem to know whether every Banach space with a $C^{p}$ smooth equivalent norm has $C^{p}$ 
smooth partitions of unity. If a positive answer to this question is ever reached, then Theorem 1.1 will be an extension of the main theorem in [4]. Otherwise and for the time being, by combining Theorem 1.1 with the main result of [4], one can easily show the following.

Corollary 1.2. Let $X$ be an infinite-dimensional Banach space. Assume that either $X$ has a $C^{p}$ smooth norm or else $X$ has $C^{p}$ smooth partitions of unity. Then, for every compact set $K \subset X$ and every $C^{p}$ smooth starlike body $A$ such that $K \subset \operatorname{int}(A)$, there exists a $C^{p}$ diffeomorphism $h: X \longrightarrow X \backslash K$ such that $h$ is the identity outside $A$.

It should be noted that, so far, no one knows of an infinite-dimensional Banach space with a $C^{1}$ bump function which does not have either a $C^{1}$ smooth norm or $C^{1}$ smooth partitions of unity (hence which does not fall into the category to which the above corollary applies). On the other hand, it is easy to see that the existence of a $C^{1}$ smooth bump is a necessary condition for a Banach space $X$ to have a diffeomorphism from $X$ onto $X \backslash\{0\}$ which restricts to the identity outside some ball.

At this point we need to introduce some terminology and notation concerning starlike bodies, which, apart from the statements of the preceding results, will play a key role in our proofs.

A closed subset $A$ of a Banach space $X$ is said to be a starlike body if there exists a point $a_{0}$ in the interior of $A$ such that every ray emanating from $a_{0}$ meets $\partial A$, the boundary of $A$, at most once. We will say that $a_{0}$ is a center of $A$. There can obviously exist many centers for a given starlike body. Up to a suitable translation, we can always assume that $a_{0}=0$ is the origin of $X$, and we will often do so, unless otherwise stated. For a starlike body $A$ with center $a_{0}$, we define the characteristic cone of $A$ as

$$
\text { cc } A=\left\{x \in X: a_{0}+r\left(x-a_{0}\right) \in A \text { for all } r>0\right\},
$$

and the Minkowski functional of $A$ with respect to the center $a_{0}$ as

$$
\mu_{A, a_{0}}(x)=\mu_{A}(x)=\inf \left\{t>0: x-a_{0} \in t\left(-a_{0}+A\right)\right\} \quad \text { for all } x \in X .
$$

Note that $\mu_{A}(x)=\mu_{-a_{0}+A}\left(x-a_{0}\right)$ for all $x \in X$. It is easily seen that $\mu_{A}$ is a continuous function which satisfies $\mu_{A}\left(a_{0}+r x\right)=r \mu_{A}\left(a_{0}+x\right)$ for every $r \geq 0$ and $x \in X$, and $\mu_{A}^{-1}(0)=$ cc $A$. Moreover, $A=\left\{x \in X: \mu_{A}(x) \leq 1\right\}$, and $\partial A=\left\{x \in X: \mu_{A}(x)=1\right\}$. Conversely, if $\psi: X \rightarrow[0, \infty)$ is continuous and satisfies $\psi\left(a_{0}+\lambda x\right)=\lambda \psi\left(a_{0}+x\right)$ for all $\lambda \geq 0$, then the set $A_{\psi}=\{x \in X: \psi(x) \leq 1\}$ is a starlike body. More generally, for a continuous function $\psi: X \rightarrow[0, \infty)$ such that $\psi_{x}(\lambda)=\psi\left(a_{0}+\lambda x\right), \lambda>0$, is increasing and $\sup \left\{\psi_{x}(\lambda): \lambda>0\right\}>\varepsilon$ for every $x \in X \backslash \psi^{-1}(0)$, the set $\psi^{-1}([0, \varepsilon])$ is a starlike body whose characteristic cone is $\psi^{-1}(0) \ni a_{0}$.

A familiar important class of starlike bodies are convex bodies, that is, starlike bodies that are convex. For a convex body $U$, cc $U$ is always a 
convex set, but in general the characteristic cone of a starlike body is not convex.

We will say that $A$ is a $C^{p}$ smooth starlike body provided its Minkowski functional $\mu_{A}$ is $C^{p}$ smooth on the set $X \backslash \operatorname{cc} A=X \backslash \mu_{A}^{-1}(0)$. This is equivalent to saying that $\partial A$ is a $C^{p}$ smooth one-codimensional submanifold of $X$ such that no affine hyperplane tangent to $\partial A$ contains a ray emanating from the center $a_{0}$. Throughout this paper, $p=0,1, \ldots, \infty$, and $C^{0}$ smooth means just continuous.

We will also say that $A$ is Lipschitz if $\mu_{A}$ is a Lipschitz function on $X$. It is easy to see that every convex body is Lipschitz with respect to any point in its interior (but this is no longer true if we drop convexity: even in the plane $\mathbb{R}^{2}$ there are starlike bodies which are not Lipschitz).

All the starlike bodies that we deal with in this paper are radially bounded. A starlike body $A$ is said to be radially bounded provided that, for every ray emanating from the center $a_{0}$ of $A$, the intersection of this ray with $A$ is a bounded set. This amounts to saying that cc $A=\left\{a_{0}\right\}$. In finite dimensions every radially bounded starlike body is in fact bounded (because the Minkowski functional of the body attains an absolute minimum on the unit sphere, which is compact), but this is no longer true in infinitedimensional Banach spaces. For instance, $A=\left\{x \in \ell_{2}: \sum_{n=1}^{\infty} x_{n}^{2} / 2^{n} \leq 1\right\}$ is a radially bounded convex body which is not bounded in the Hilbert space $\ell_{2}$; the body $A$ is the unit ball of the nonequivalent $C^{\infty}$ smooth norm $\omega(x)=\left(\sum_{n=1}^{\infty} x_{n}^{2} / 2^{n}\right)^{1 / 2}$ in $\ell_{2}$. For every bounded starlike body $A$ in a Banach space $(X,\|\cdot\|)$ there are constants $M, m>0$ such that $m\|x\| \leq$ $\mu_{A}(x) \leq M\|x\|$ for all $x \in X$. If $A$ is just radially bounded then we can only ensure that $\mu_{A}(x) \leq M\|x\|$ for all $x \in X$, for some $M>0$. As is shown implicitly in [14, Proposition II.5.1], a Banach space $X$ has a $C^{p}$ smooth bump function if and only if there is a bounded $C^{p}$ smooth starlike body in $X$.

We finish these preliminaries with some nonstandard notation concerning strict inclusions between starlike bodies. In our proofs we will often require that, for a couple of starlike bodies $A \subset B$, the boundaries of $A$ and $B$ are well separated. There are at least two nonequivalent natural notions of separation between boundaries of starlike bodies, and we will need to use both of them, as each one has its own advantages. The strongest and most natural notion corresponds to the fact that the distance between $A$ and $X \backslash B$ is positive. We will use the notation $A \subset_{\mathrm{d}} B$ to mean that $\operatorname{dist}(A, X \backslash B)>0$, and we will say that $B$ strictly contains $A$ in the distance sense. Notice that this notion makes sense even though $A$ and $B$ do not have the same center, or even if $A$ and $B$ are any sets, not necessarily starlike.

The other useful notion is that the Minkowski functionals of $A$ and $B$ are well separated, in the following sense. First, note that if $A \subseteq B$ are starlike 
with respect to the same center $a_{0}$ then we always have $\mu_{B}(x) \leq \mu_{A}(x)$ for all $x \in X$. If we also know that $\sup _{x \in A} \mu_{B}(x)<1$ then we will write $A \subset_{\mu} B$, saying that $B$ strictly contains $A$ in the gauge sense. This is equivalent to saying that there exists some $\delta>0$ such that $a_{0}+(1+\delta)\left(-a_{0}+A\right) \subseteq B$. Of course, this notion only makes sense when $A$ and $B$ have at least one center $a_{0}$ in common. It is immediate to see that $A \subset_{\mathrm{d}} B$ implies that $A \subset_{\mu} B$. The converse is false in general, unless $A$ is Lipschitz. When $A \subset B$ have the same center and $A$ is Lipschitz we have $A \subset_{\mathrm{d}} B$ if and only if $A \subset_{\mu} B$ (see Lemma 2.6 below).

2. Proof of the main result. In contrast with Bessaga-type constructions $[8,15,1,4,5]$, our proof does not provide an explicit elegant formula for the deleting diffeomorphism. We rather turn to the origins and find inspiration in the geometrical ideas of the pioneering work of Klee [20] (see also [23]). We will need to consider an infinite composition of carefully constructed self-diffeomorphisms of $X$.

The main ingredient of our proof is the following proposition, which implies that if our infinite-dimensional space $X$ has enough smooth starlike bodies then every weakly compact set $K$ can be removed by means of a diffeomorphism $h: X \rightarrow X \backslash K$ which is the identity outside some starlike body.

Proposition 2.1. Let $X$ be a Banach space, and $K$ a subset of $X$. Assume that there are sequences $\left(P_{n}\right),\left(C_{n}\right),\left(A_{n}\right),\left(B_{n}\right),\left(Q_{n}\right),\left(D_{n}\right),\left(E_{n}\right)$ of subsets of $X$ and a sequence $\left(c_{n}\right)$ of points of $X$ satisfying the following conditions for each $n \in \mathbb{N}$ :

(1) $A_{n}, B_{n}, Q_{n}, D_{n}, E_{n}$ are radially bounded $C^{p}$ smooth starlike bodies with respect to $c_{n+2}$;

(2) $C_{n+2} \subset D_{n} \subset_{\mu} E_{n} \subset_{\mu} A_{n} \subset C_{n+1} \subset P_{n+1} \subset B_{n} \subset_{\mu} Q_{n} \subset P_{n}$;

(3) $\bigcap_{n=1}^{\infty} C_{n}=\emptyset$,

(4) $\bigcap_{n=1}^{\infty} P_{n}=K$.

Then there exists a $C^{p}$ diffeomorphism $\Psi: X \rightarrow X \backslash K$ such that $\Psi$ is the identity on $X \backslash P_{1}$.

In order to prove this proposition we will only require a simple geometrical lemma. The purely topological version of this result is very easy (see $[12,23]$, where the authors do not even bother to write the formulas), but the smooth case is a little more difficult and requires a proof.

LEMma 2.2 (The Four Bodies Lemma). Let $X$ be a Banach space, and let $A, B, C, D$ be four radially bounded $C^{p}$ smooth starlike bodies with respect to the same point $a_{0} \in \operatorname{int}(A)$. Assume that

$$
A \subset_{\mu} B \subset C \subset_{\mu} D .
$$


Then there exists a $C^{p}$ diffeomorphism $h: X \rightarrow X$ such that

(1) $h(B)=C$,

(2) $h$ is the identity on $A \cup(X \backslash D)$.

Proof. We may assume $a_{0}=0$. Since $A \subset_{\mu} B$ and $C \subset_{\mu} D$, there exists some $\delta \in(0,1)$ such that $A \subset(1-\delta) B$ and $(1+\delta) C \subset D$. Take a $C^{\infty}$ smooth function $\lambda: \mathbb{R} \rightarrow \mathbb{R}$ such that $\lambda$ is nondecreasing, $\lambda(t)=0$ if $t \leq 1-\delta$, and $\lambda(t)=1$ for $t \geq 1$. Define then $f: X \rightarrow X$ by

$$
f(x)=\left[\lambda\left(\mu_{B}(x)\right) \frac{\mu_{B}(x)}{\mu_{C}(x)}+1-\lambda\left(\mu_{B}(x)\right)\right] x \quad \text { if } x \neq 0,
$$

and $f(0)=0$. It is easy to check that $f$ is a $C^{p}$ diffeomorphism of $X$ such that $f(B)=C$ and $f$ is the identity on $A$.

On the other hand, pick a $C^{\infty}$ smooth function $\theta: \mathbb{R} \rightarrow \mathbb{R}$ such that $\theta$ is nonincreasing, $\theta(t)=1$ if $t \leq 1+\delta / 4$, and $\theta(t)=0$ if $t \geq 1+\delta / 2$. Consider the mapping $g: X \backslash\{0\} \rightarrow X \backslash\{0\}$ defined by

$$
g(x)=\left[\theta\left(\mu_{C}(x)\right) \frac{\mu_{C}(x)}{\mu_{B}(x)}+1-\theta\left(\mu_{C}(x)\right)\right] x,
$$

which is a $C^{p}$ diffeomorphism as well. Now define $h: X \rightarrow X$ by

$$
h(x)= \begin{cases}f(x) & \text { if } \mu_{B}(x)<1+\delta / 4, \\ g^{-1}(x) & \text { if } 1<\mu_{B}(x) .\end{cases}
$$

Observe that if $1 \leq \mu_{B}(x) \leq 1+\delta / 4$ then $f(x)=\left[\mu_{B}(x) / \mu_{C}(x)\right] x=g^{-1}(x)$; hence $h$ is well defined and locally a $C^{p}$ diffeomorphism. Moreover, it is easy to see that $h(X \backslash(1+\delta / 4) B)=X \backslash(1+\delta / 4) C$, which (bearing in mind the definition of $h$ ) implies that $h$ is one-to-one. On the other hand, since $h((1+\delta / 4) B)=(1+\delta / 4) C$ and $h(X \backslash B)=g^{-1}(X \backslash B)=X \backslash C$, it follows that $h$ is a surjection. Therefore $h: X \rightarrow X$ is a $C^{p}$ diffeomorphism. Finally, it is clear that $h(B)=C$, and $h$ is the identity on $A \cup(X \backslash(1+\delta / 2) B) \supset$ $A \cup(X \backslash D)$.

Proof of Proposition 2.1. The proof of this proposition, as well as some parts of that of Proposition 2.3 below, resemble the arguments included in [23] (which in turn are inspired, like the rest of the present paper, by Klee's seminal work [20]).

Fix any $n \in \mathbb{N}$. Consider the inclusions of bodies

$$
D_{n} \subset_{\mu} E_{n} \subset B_{n} \subset_{\mu} Q_{n}, \quad D_{n} \subset_{\mu} E_{n} \subset A_{n} \subset_{\mu} Q_{n} .
$$

According to the Four Bodies Lemma there exist $C^{p}$ diffeomorphisms $f_{n}, g_{n}$ : $X \rightarrow X$ such that

$$
\begin{array}{ll}
f_{n}\left(E_{n}\right)=B_{n}, & f_{n} \text { is the identity on } D_{n} \cup\left(X \backslash Q_{n}\right), \\
g_{n}\left(E_{n}\right)=A_{n}, & g_{n} \text { is the identity on } D_{n} \cup\left(X \backslash Q_{n}\right) .
\end{array}
$$


Define then $h_{n}=g_{n} \circ f_{n}^{-1}: X \rightarrow X$, which is a $C^{p}$ diffeomorphism of $X$ such that

$$
h_{n}\left(B_{n}\right)=A_{n}, \quad h_{n} \text { is the identity on } D_{n} \cup\left(X \backslash Q_{n}\right) .
$$

Now consider the family $\left(h_{n}\right)$ of $C^{p}$ diffeomorphisms. For each $n \in \mathbb{N}$ define the mapping $\psi_{n}: X \rightarrow X$ to be the composition

$$
\psi_{n}(x)=\left(h_{1} \circ \ldots \circ h_{n}\right)(x),
$$

which is obviously a $C^{p}$ diffeomorphism of $X$. Since $h_{n}$ is the identity on $X \backslash Q_{n}$ and $Q_{n} \subset P_{n}$, we see that $h_{n}$ is the identity on $X \backslash P_{n}$. It follows that

$$
\left.\psi_{n}\right|_{X \backslash P_{n}}=\left.\psi_{n-1}\right|_{X \backslash P_{n}} \quad \text { for all } n \geq 2 .
$$

Note that, from the conditions in the statement of Proposition 2.1, we know that

$$
X \backslash P_{n} \subset X \backslash P_{n+1} \subset X \backslash K \text { for all } n, \quad X \backslash K=\bigcup_{n=1}^{\infty} X \backslash P_{n} .
$$

Then we can define $\psi: X \backslash K \rightarrow X$ by letting

$$
\left.\psi\right|_{X \backslash P_{n+1}}=\left.\psi_{n}\right|_{X \backslash P_{n+1}} \quad \text { for each } n \in \mathbb{N} .
$$

Taking equations (1) and (2) above into account, it is clear that the mapping $\psi$ is well defined, one-to-one, and is locally a $C^{p}$ diffeomorphism. Let us see that $\psi$ is surjective and therefore a $C^{p}$ diffeomorphism from $X \backslash K$ onto $X$.

Bearing in mind that $h_{j}$ is the identity on $D_{j} \supset C_{j+2}$ and $A_{j} \subset C_{j+1}$, we have $h_{j}\left(A_{n}\right)=A_{n}$ if $j \leq n-1$, and since $h_{n}\left(B_{n}\right)=A_{n}$ we may deduce that

$$
\begin{aligned}
\psi_{n}\left(B_{n}\right)=h_{1} \circ \ldots \circ h_{n}\left(B_{n}\right) & =h_{1} \circ \ldots \circ h_{n-1}\left(A_{n}\right) \\
& =h_{1} \circ \ldots \circ h_{n-2}\left(A_{n}\right)=\ldots=A_{n},
\end{aligned}
$$

and in particular $\psi_{n}\left(X \backslash B_{n}\right)=X \backslash A_{n}$. But, by the hypothesis on the bodies, $P_{n+1} \subset B_{n} \subset P_{n}$, that is, $X \backslash P_{n} \subset X \backslash B_{n} \subset X \backslash P_{n+1}$, and hence

$$
\psi\left(X \backslash B_{n}\right)=\psi_{n}\left(X \backslash B_{n}\right)=X \backslash A_{n} .
$$

Now, note that the hypotheses of Proposition 2.1 imply that $C_{n+2} \subset A_{n} \subset$ $C_{n+1}, \bigcap_{n=1}^{\infty} C_{n}=\emptyset$, which yields

$$
X=\bigcup_{n=1}^{\infty}\left(X \backslash A_{n}\right)
$$

On the other hand, since $K=\bigcap_{n=1}^{\infty} P_{n+1} \subset \bigcap_{n=1}^{\infty} B_{n} \subset \bigcap_{n=1}^{\infty} P_{n}=K$, we have

$$
X \backslash K=\bigcup_{n=1}^{\infty}\left(X \backslash B_{n}\right) \text {. }
$$


Now, by combining equations (4), (5) and (6), we get

$$
\psi(X \backslash K)=\psi\left(\bigcup_{n=1}^{\infty}\left(X \backslash B_{n}\right)\right)=\bigcup_{n=1}^{\infty}\left(X \backslash A_{n}\right)=X,
$$

hence $\psi$ is a $C^{p}$ diffeomorphism from $X \backslash K$ onto $X$. Moreover, if $x \in$ $X \backslash P_{1} \subset X \backslash P_{2}$, from the definition of $\psi$, and bearing in mind that $h_{1}$ is the identity on $X \backslash P_{1}$, we conclude that $\psi(x)=\psi_{1}(x)=h_{1}(x)=x$. Finally, if we define $\Psi=\psi^{-1}$, it is clear that $\Psi$ is a $C^{p}$ diffeomorphism from $X$ onto $X \backslash K$ which is the identity off $P_{1}$.

The next step in the proof of our main theorem is of course to ensure that if an infinite-dimensional Banach space $X$ has $C^{p}$ smooth partitions of unity then, for every weakly compact set $K \subset X$, there are families of $C^{p}$ smooth starlike bodies satisfying the conditions of Proposition 2.1.

Proposition 2.3. Let $X$ be an infinite-dimensional Banach space which admits $C^{p}$ smooth partitions of unity. There exists $B$, a radially bounded $C^{p}$ smooth starlike body with respect to the origin, such that, for every weakly compact set $K \subset X$ and every $r>0$ such that $K \subset r B$, there are sequences $\left(P_{n}\right),\left(C_{n}\right),\left(A_{n}\right),\left(B_{n}\right),\left(Q_{n}\right),\left(D_{n}\right),\left(E_{n}\right)$ of subsets of $X$ and a sequence $\left(c_{n}\right)$ of points of $X$ satisfying the following conditions for each $n \in \mathbb{N}$ :

(1) $A_{n}, B_{n}, Q_{n}, D_{n}, E_{n}$ are radially bounded $C^{p}$ smooth starlike bodies with respect to $c_{n+2}$;

(2) $C_{n+2} \subset D_{n} \subset_{\mu} E_{n} \subset_{\mu} A_{n} \subset C_{n+1} \subset P_{n+1} \subset B_{n} \subset_{\mu} Q_{n} \subset P_{n}$;

(3) $\bigcap_{n=1}^{\infty} C_{n}=\emptyset$;

(4) $\bigcap_{n=1}^{\infty} P_{n}=K$

(5) $P_{1} \subset 4 r B$.

The proof of Proposition 2.3 is quite long and will be split into several lemmas.

Notation 2.4. If $X$ is a Banach space and $B_{X}=\{x \in X:\|x\| \leq 1\}$ is its unit ball, for all subsets $A, B$ of $X$ and for every $\varepsilon>0$, we define

$$
[A, B]=\{t x+(1-t) y: x \in A, y \in B, t \in[0,1]\},
$$

and $N(A, \varepsilon)=\{x \in X: \operatorname{dist}(x, A) \leq \varepsilon\}=\overline{A+\varepsilon B_{X}}$. When $A=\{a\}$ is a singleton we will simply write $[A, B]=[a, B]$.

Lemma 2.5. Let $X$ be a Banach space, $C$ a bounded convex body in $X$, and $K$ a weakly compact subset of $X$. Then $V:=[K, C]$ is a starlike body with respect to every interior point of $C$. Moreover, $V$ is bounded and $\mu_{V}$ : $X \rightarrow[0, \infty)$ is Lipschitz.

Proof. Since $C \subseteq V$, it is obvious that $V$ has nonempty interior. By using the (weak) compactness of $K$ and $[0,1]$, it is easy to see that $V$ is closed. 
Now let us see that $V$ is starlike with respect to every point $x_{0} \in \operatorname{int}(C)$. Take two points $x_{1}, x_{2} \in \partial V \subset V$ with $x_{1} \in\left[x_{0}, x_{2}\right]$. Assuming that $x_{1} \neq x_{2}$ we will get a contradiction. Indeed, since $V=\bigcup_{y \in K}[y, C]$ and $x_{1} \in \partial V$, we have $x_{1} \in X \backslash \operatorname{int}([y, C])$ for every $y \in K$. Hence, for every $y \in K$, either $x_{1} \in \partial[y, C]$ or $x_{1} \notin[y, C]$; in either case, since $[y, C]$ is a starlike body with respect to $x_{0} \in \operatorname{int}(C)$, and $x_{2} \neq x_{1} \in\left[x_{0}, x_{2}\right]$, we deduce that $x_{2} \notin[y, C]$. But then $x_{2} \notin \bigcup_{y \in K}[y, C]=V$, a contradiction.

It is obvious that $V$ is bounded. It only remains to show that $\mu_{V}$ (with respect to any point $x_{0} \in \operatorname{int}(C)$ ) is Lipschitz. Without loss of generality we may assume that the given center is $x_{0}=0$. Let $M>0$ be such that $\mu_{C}(x) \leq$ $M\|x\|$ for all $x \in X$. Since $C \subseteq[y, C]$ we have $\mu_{[y, C]}(x) \leq \mu_{C}(x) \leq M\|x\|$ for all $x \in X$ and, bearing in mind that $[y, C]$ is a convex body, this means that $\mu_{[y, C]}$ is $M$-Lipschitz for all $y \in K$. On the other hand, it is easily seen that $\mu_{V}(x)=\inf _{y \in K} \mu_{[y, C]}(x)$. Since the infimum of $M$-Lipschitz functions is always an $M$-Lipschitz function, we see that $\mu_{V}$ is $M$-Lipschitz.

LEMMA 2.6. Let $X$ be a Banach space, and $A$ a Lipschitz starlike body with respect to the origin. Then, for every $\varepsilon>0$, there exists $\delta>0$ so that $A+\delta B_{X} \subset(1+\varepsilon) A$.

Proof. Let $M$ be a Lipschitz constant for $\mu_{A}$. For a given $\varepsilon>0$ choose $\delta>0$ with $\delta M<\varepsilon$. Take $x=y+z$, with $y \in A, z \in \delta B_{X}$. Then we have $\mu_{A}(x)=\mu_{A}(y+z)-\mu_{A}(y)+\mu_{A}(y) \leq M\|z\|+\mu_{A}(y) \leq M \delta+1<1+\varepsilon$.

LEMma 2.7. Let $C$ be a bounded convex body in a Banach space $X$, with $0 \in \operatorname{int}(C)$. Then, for every $\delta \in(0,1)$, $\operatorname{dist}((1-\delta) C, X \backslash C)>0$, that is, $(1-\delta) C \subset_{\mathrm{d}} C$.

Proof. This is an easy consequence of the preceding lemma and the fact that $\mu_{C}$ is Lipschitz because $C$ is a convex body.

Lemma 2.8. Let $T: X \rightarrow Y$ be a continuous linear injection between two Banach spaces. Then, for every radially bounded $C^{p}$ smooth body $B^{\prime}$ in $Y$ which is starlike with respect to a point $b^{\prime} \in T(X)$, the set $B=T^{-1}\left(B^{\prime}\right)$ is a radially bounded $C^{p}$ smooth starlike body in $X$ with respect to $b=$ $T^{-1}\left(b^{\prime}\right)$.

Proof. Let $b^{\prime}=T(b)$ be the center of $B^{\prime}$. Then $A^{\prime}:=-b^{\prime}+B^{\prime}$ is starlike with respect to the origin, radially bounded and $C^{p}$ smooth. Consider the function $\psi: X \rightarrow[0, \infty)$ defined by $\psi(x)=\mu_{A^{\prime}}(T(x))$. Then $A:=\{x \in X:$ $\psi(x) \leq 1\}$ is a $C^{p}$ smooth starlike body in $X$ (with respect to the origin); besides, since $\psi(x)>0$ whenever $x \neq 0$, we have cc $A=\{0\}$, that is, $A$ is radially bounded. It is obvious that $A=T^{-1}\left(A^{\prime}\right)$. Then $B=T^{-1}\left(B^{\prime}\right)=$ $T^{-1}\left(b^{\prime}+A^{\prime}\right)=b+A$ is a radially bounded $C^{p}$ smooth starlike body with respect to $b \in X$. 
Lemma 2.9. Let $T: X \rightarrow Y$ be a continuous linear injection between two Banach spaces. Assume that $A^{\prime}$ and $B^{\prime}$ are starlike bodies with respect to $y_{0}=T\left(x_{0}\right) \in T(X)$, and $A^{\prime} \subset_{\mu} B^{\prime}$. Then $A:=T^{-1}\left(A^{\prime}\right) \subset_{\mu} T^{-1}\left(B^{\prime}\right):=B$.

Proof. Left to the reader.

The following lemmas show how one can approximate and interpolate starlike bodies with smooth starlike bodies, provided the space has smooth partitions of unity.

LEMMA 2.10. Let $X$ be a Banach space with $C^{p}$ smooth partitions of unity, and $C$ a starlike body with $\operatorname{cc} C=\{0\}$. Then, for every $\delta>0$, there exists $A \subset X$, a $C^{p}$ smooth starlike body, with cc $A=\{0\}$ and such that $(1-\delta) C \subset A \subset(1+\delta) C$.

Proof. Since $X$ has $C^{p}$ smooth partitions of unity, it has a $C^{p}$ smooth bump as well, and in particular there exists a bounded $C^{p}$ smooth starlike body $B$ with respect to the origin [14, Proposition II.5.1]. Choose $\varepsilon_{0} \in(0,1)$ such that $1 /\left(1-\varepsilon_{0}\right)<1+\delta$, and $1+\varepsilon_{0}<1 /(1-\delta)$. Define $\varepsilon: X \backslash\{0\} \rightarrow$ $(0, \infty)$ by $\varepsilon(x)=\varepsilon_{0} \mu_{C}(x)$ for all $x \neq 0$, which is a continuous strictly positive function. Since $X$ has $C^{p}$ smooth partitions of unity, so does its open subset $X \backslash\{0\}$, and therefore every continuous function on $X \backslash\{0\}$ can be $\varepsilon$-approximated by a $C^{p}$ smooth function on $X \backslash\{0\}$. Hence, given the continuous function $\mu_{C}: X \backslash\{0\} \rightarrow(0, \infty)$, there exists a $C^{p}$ smooth function $g: X \backslash\{0\} \rightarrow \mathbb{R}$ such that $\left|\mu_{C}(x)-g(x)\right| \leq \varepsilon(x)$ for all $x \neq 0$. Now define $\psi: X \rightarrow \mathbb{R}$ by

$$
\psi(x)=\mu_{B}(x) g\left(\frac{x}{\mu_{B}(x)}\right) \quad \text { if } x \neq 0,
$$

and $\psi(0)=0$. The function $\psi$ is clearly continuous on $X$, of class $C^{p}$ on $X \backslash\{0\}$, and positively homogeneous. Moreover,

$$
\begin{aligned}
& \left|\psi(x)-\mu_{C}(x)\right|=\left|\mu_{B}(x) g\left(\frac{x}{\mu_{B}(x)}\right)-\mu_{C}(x)\right| \\
& \quad=\left|\mu_{B}(x) g\left(\frac{x}{\mu_{B}(x)}\right)-\mu_{B}(x) \mu_{C}\left(\frac{x}{\mu_{B}(x)}\right)\right| \leq \mu_{B}(x) \varepsilon\left(\frac{x}{\mu_{B}(x)}\right)=\varepsilon_{0} \mu_{C}(x)
\end{aligned}
$$

for all $x \neq 0$. In particular, $\psi(x) \geq\left(1-\varepsilon_{0}\right) \mu_{C}(x)>0$ if $x \neq 0$. Therefore, $A:=\{x \in X: \psi(x) \leq 1\}$ is a $C^{p}$ smooth starlike body with respect to 0 . Let us check that $A$ approximates $C$ as required. We have

$$
\begin{aligned}
x \in A & \Leftrightarrow \psi(x) \leq 1 \Rightarrow \mu_{C}(x) \leq 1+\varepsilon_{0} \mu_{C}(x) \Rightarrow\left(1-\varepsilon_{0}\right) \mu_{C}(x) \leq 1 \\
& \Rightarrow x \in \frac{1}{1-\varepsilon_{0}} C \subset(1+\delta) C,
\end{aligned}
$$

so $A \subset(1+\delta) C$. On the other hand, if $x \in(1-\delta) C$, that is, $\mu_{C}(x) \leq 1-\delta$, then $\psi(x) \leq\left(1+\varepsilon_{0}\right) \mu_{C}(x) \leq\left(1+\varepsilon_{0}\right)(1-\delta)<1$, hence $x \in A$. 
Lemma 2.11. Let $X$ be a Banach space with $C^{p}$ smooth partitions of unity, $K$ a closed subset of $X$, and $D$ a bounded starlike body with respect to 0 such that $K \subset_{\mathrm{d}} D$. Then there exist $C^{p}$ smooth starlike bodies $D_{1}$ and $D_{2}$ with respect to 0 such that

$$
K \subset D_{1} \subset_{\mu} D_{2} \subset D .
$$

Moreover, if $K$ is a bounded starlike body with respect to 0 , the above is true for any set $D$, and the starlike body $D_{2}$ satisfies $D_{2} \subset_{\mathrm{d}} D$.

Proof. Since $K \subset_{\mathrm{d}} D$ we can take $0<\theta<1 / 2$ so that $K \subset(1-2 \theta) D$. Choose $\delta \in(0,1)$ with $(1-2 \theta) /(1-\theta)<1-\delta$ and $(1+\delta)(1-\theta)<1$. Applying the preceding lemma to $C:=(1-\theta) D$, we get a $C^{p}$ smooth starlike body $D_{1}$ with respect to 0 such that $(1-\delta) C \subset D_{1} \subset(1+\delta) C$. In particular, taking into account that $1-2 \theta<(1-\theta)(1-\delta)$, we deduce $K \subset(1-2 \theta) D \subset(1-\theta)(1-\delta) D=(1-\delta) C \subset D_{1}$. Now pick $\varepsilon>0$ such that $(1+\varepsilon)(1+\delta)(1-\theta)<1$, and set $D_{2}:=(1+\varepsilon) D_{1}$. The body $D_{2}$ is $C^{p}$ smooth and starlike with respect to 0 , and $D_{1} \subset{ }_{\mu} D_{2}$. Finally, we also have $D_{2}=(1+\varepsilon) D_{1} \subset(1+\varepsilon)(1+\delta) C \subset(1+\varepsilon)(1+\delta)(1-\theta) D \subset D$.

Assume that $K$ is a bounded starlike body with respect to 0 , and $D$ is any subset of $X$ such that $K \subset_{\mathrm{d}} D$. Choose real numbers $\varepsilon>0$ and $\delta \in(0,1)$ satisfying $1<(1-\delta)(1+\varepsilon)$ and $(1+\delta)(1+\varepsilon) K \subset_{\mathrm{d}} D$. By imitating the previous paragraph, with $C:=(1+\varepsilon) K$, we obtain $D_{1}$, a $C^{p}$ smooth starlike body with respect to 0 , such that $(1-\delta) C \subset D_{1} \subset(1+\delta) C$. Bearing in mind the choice of $\delta$ and $\varepsilon$, we deduce that $K \subset D_{1} \subset_{\mathrm{d}} D$. Now it is clear how to define $D_{2}$, a $C^{p}$ smooth starlike body with respect to 0 such that $D_{1} \subset_{\mu} D_{2} \subset_{\mathrm{d}} D$.

The following lemma is one of the keys to the proof of Proposition 2.3.

Lemma 2.12. Let $X$ be a nonreflexive Banach space, $K$ a weakly compact set, and $C$ a bounded convex body with $0 \in \operatorname{int}(C)$ and $K \subset_{\mathrm{d}} C$. Then there exist $\varepsilon>0$ and a sequence $\left(C_{n}\right)$ of convex bodies such that

(1) $\bigcap_{n=1}^{\infty} C_{n}=\emptyset$,

(2) $C_{n+1} \subset_{\mathrm{d}} C_{n} \subset C$ for all $n \in \mathbb{N}$,

(3) $\left[K, C_{1}\right]+3 \varepsilon B_{X} \subset C$.

Proof. Since $K \subset_{\mathrm{d}} C$, there exists $\delta_{0}>0$ such that $K \subset\left(1-2 \delta_{0}\right) C$ and, by Lemma 2.7 , $\operatorname{dist}\left(\left(1-\delta_{0}\right) C, X \backslash C\right) \geq \delta_{1}$ for some $\delta_{1}>0$.

Since $X$ is nonreflexive, according to James's theorem, there exists a continuous linear functional $T \in X^{*}$ such that $T$ does not attain its supremum on the body $\left(1-2 \delta_{0}\right) C$; let $\alpha$ be that supremum. Define now $H_{n}:=$ $\left\{x \in\left(1-2 \delta_{0}\right) C: T(x) \geq \alpha-1 / n\right\}$ for each $n \in \mathbb{N}$. We have $\bigcap_{n=1}^{\infty} H_{n}=\emptyset$, $H_{n+1} \subset H_{n}$ for all $n$, and $H_{1} \subset\left(1-2 \delta_{0}\right) C \subset_{\mathrm{d}}\left(1-\delta_{0}\right) C$. Take $\varepsilon>0$ such 
that $H_{1}+\varepsilon B_{X} \subset\left(1-\delta_{0}\right) C$ and $3 \varepsilon<\delta_{1}$. Then for each $n \in \mathbb{N}$ define

$$
C_{n}=N\left(H_{n}, \varepsilon / 2^{n}\right)=\left\{x \in X: \operatorname{dist}\left(x, H_{n}\right) \leq \varepsilon / 2^{n}\right\} .
$$

It is easy to see that $\left(C_{n}\right)$ has the three properties of the statement.

Proof of Proposition 2.3

CASE I: $X$ is nonreflexive. Let $E$ be a bounded convex body with $0 \in$ $\operatorname{int}(E)$. By Lemma 2.7, we have $(1 / 8) E \subset_{\mathrm{d}}(1 / 4) E \subset_{\mathrm{d}}(1 / 2) E$. According to Lemma 2.10, there exists a $C^{p}$ smooth starlike body with respect to 0 such that $(1 / 8) E \subset B \subset(1 / 4) E$. This body $B$ is the one we need.

Now take a weakly compact set $K \subset X$ such that $K \subset r B$. Hence $K \subset r B \subset(r / 4) E \subset_{\mathrm{d}}(r / 2) E$. According to Lemma 2.12, there exists $\varepsilon>0$ and a sequence $\left(C_{n}\right)$ of convex bodies such that

$$
\bigcap_{n=1}^{\infty} C_{n}=\emptyset, \quad\left[K, C_{1}\right]+3 \varepsilon B_{X} \subset(r / 2) E, \quad C_{n+1} \subset_{\mathrm{d}} C_{n} \subset r E \quad \text { for all } n \in \mathbb{N} .
$$

Let us choose a sequence $\left(c_{n}\right)$ of points of $X$ such that $c_{n} \in \operatorname{int}\left(C_{n}\right)$ for every $n \in \mathbb{N}$. Set $\Delta=\operatorname{diam}((r / 2) E)>0$. For each $n \in \mathbb{N}$, define

$$
V_{n}=\left[C_{n}, K\right] \text {. }
$$

By Lemma 2.5, $V_{n}$ is a Lipschitz starlike body with respect to every point in the interior of $C_{n}$. Let $\mu_{n}=\mu_{V_{n}}$ be the Minkowski functional of $V_{n}$ with respect to the point $c_{n+1} \in \operatorname{int}\left(C_{n+1}\right) \subset \operatorname{int}\left(C_{n}\right)$. Note that $\mu_{n}$ is a Lipschitz function.

Next we are going to inductively construct a sequence $\left(\delta_{n}\right)$ of positive numbers such that, if we define

$$
P_{n}:=\left\{x \in X: \mu_{n}(x) \leq 1+\delta_{n}\right\}
$$

for each $n \in \mathbb{N}$, then $\left(P_{n}\right)$ is a sequence of bounded starlike bodies such that

(i) $P_{n+1} \subset_{\mathrm{d}} P_{n} \subset P_{1} \subset(r / 2) E$ for all $n \in \mathbb{N}$,

(ii) $\bigcap_{n=1}^{\infty} P_{n}=K$,

(iii) $P_{n}$ is starlike with respect to $c_{n+1}$ for all $n \in \mathbb{N}$,

(iv) $C_{n+1} \subset_{\mathrm{d}} P_{n} \cap C_{n}$ for all $n \in \mathbb{N}$.

SteP 1. Choose $\delta_{1}>0$ with $\delta_{1}<\min \{\varepsilon / \Delta, 1\}$, and set $P_{1}=\{x \in X$ : $\left.\mu_{1}(x) \leq 1+\delta_{1}\right\}$. By Lemma 2.6, there is $\delta_{1}^{\prime}>0$ such that $P_{1} \supset V_{1}+\delta_{1}^{\prime} B_{X}$.

STEP 2. Now choose $\delta_{2}>0$ such that $\delta_{2}<\min \left\{\delta_{1}^{\prime} / 2 \Delta, 1 / 2\right\}$. Then $P_{2}=\left\{x \in X: \mu_{2}(x) \leq 1+\delta_{2}\right\} \subset V_{2}+\left(\delta_{1}^{\prime} / 2\right) B_{X}$, and so $\operatorname{dist}\left(P_{2}, X \backslash P_{1}\right)>0$.

StEP $(n+1)$. Assume $\delta_{j}$ and $P_{j}$ are already defined for $j=1, \ldots, n$ in such a way that $P_{j+1} \subset_{\mathrm{d}} P_{j}$ for $j \leq n-1$. By Lemma 2.6, there is $\delta_{n}^{\prime}>0$ such that $P_{n} \supset V_{n}+\delta_{n}^{\prime} B_{X}$. Pick $\delta_{n+1}>0$ so that $\delta_{n+1}<\min \left\{\delta_{n}^{\prime} / 2 \Delta, 1 / 2^{n}\right\}$, and set $P_{n+1}=\left\{x \in X: \mu_{n+1}(x) \leq 1+\delta_{n+1}\right\}$. Then $P_{n+1} \subset V_{n}+\left(\delta_{n}^{\prime} / 2\right) B_{X}$, hence $\operatorname{dist}\left(P_{n+1}, X \backslash P_{n}\right)>0$. 
By induction the sequence $\left(P_{n}\right)$ is well defined and has properties (i) and (iii) above. To see that $P_{1} \subset_{\mathrm{d}}(r / 2) E$, just note that $P_{1} \subset V_{1}+\delta_{1} \Delta B_{X}=$ $\left[C_{1}, K\right]+\delta_{1} \Delta B_{X} \subset\left[C_{1}, K\right]+3 \varepsilon B_{X} \subset(r / 2) E$. On the other hand, since $P_{n} \cap C_{n}=C_{n}$, it is clear that $C_{n+1} \subset_{\mathrm{d}} P_{n} \cap C_{n}$, that is, the sequence $\left(P_{n}\right)$ has property (iv).

Finally, let us check that condition (ii) holds as well. It is immediate that $K \subset \bigcap_{n=1}^{\infty} P_{n}$. Let us take $q \in \bigcap_{n=1}^{\infty} P_{n}$ and show that $q \in K$. For each $n \in \mathbb{N}$ we have $q \in P_{n} \subset V_{n}+\delta_{n} \Delta B_{X}=\left[C_{n}, K\right]+\delta_{n} \Delta B_{X}$, so there are $x_{n} \in C_{n}, y_{n} \in K, t_{n} \in[0,1]$ with $\left\|q-\left(1-t_{n}\right) x_{n}-t_{n} y_{n}\right\| \leq \delta_{n} \Delta$, and in particular $\lim _{n \rightarrow \infty}\left[\left(1-t_{n}\right) x_{n}-t_{n} y_{n}\right]=q$. Since $K$ is weakly compact and $[0,1]$ is compact, we may assume (passing to a subsequence if necessary) that $y_{n}$ converges to some $y_{0} \in K$ weakly, and $t_{n} \rightarrow t_{0} \in[0,1]$. Then $\left(1-t_{n}\right) x_{n}$ converges to $q-t_{0} y_{0}$ weakly. If $t_{0} \neq 1$ then $x_{n}$ converges weakly to $x_{0}:=$ $\left(1-t_{0}\right)^{-1}\left(q-t_{0} y_{0}\right)$; but, since each $C_{n} \supset\left(x_{j}\right)_{j \geq n}$ is closed and convex, hence weakly closed, we have $x_{0} \in C_{n}$ for each $n$, and then $x_{0} \in \bigcap_{n=1}^{\infty} C_{n}=\emptyset$, a contradiction. Therefore, $t_{0}=1$, and $q=y_{0} \in K$.

Now we are going to define the bodies $A_{n}, B_{n}, D_{n}, E_{n}$, and $Q_{n}$. Fix $n \in \mathbb{N}$. Since $C_{n+2}$ and $C_{n+1}$ are bounded starlike bodies with respect to $c_{n+2}$, and $C_{n+2} \subset_{\mathrm{d}} C_{n+1}$, we can apply Lemma 2.11 to obtain two $C^{p}$ smooth starlike bodies $D_{n}, E_{n}$ with respect to $c_{n+2}$ such that

$$
C_{n+2} \subset D_{n} \subset_{\mu} E_{n} \subset_{\mathrm{d}} C_{n+1} .
$$

Another application of Lemma 2.11 gives us a $C^{p}$ smooth starlike body $A_{n}$ with respect to $c_{n+2}$ such that

$$
E_{n} \subset_{\mu} A_{n} \subset C_{n+1}=C_{n+1} \cap P_{n+1} .
$$

Moreover, $P_{n+1} \subset_{\mathrm{d}} P_{n}$, and $P_{n+1}$ is starlike with respect to $c_{n+1}$. Then, applying Lemma 2.11 for the last time (now $P_{n}$ acts as a mere set, it is not necessary that $P_{n}$ be starlike with respect to $c_{n+2}$, only $P_{n+1}$ has to satisfy this condition), we get $B_{n}$ and $Q_{n}$, two $C^{p}$ smooth starlike bodies with respect to $c_{n+2}$, satisfying

$$
P_{n+1} \subset_{\mu} B_{n} \subset_{\mu} Q_{n} \subset P_{n} .
$$

Moreover, we also have $E_{n} \subset C_{n+1} \subset P_{n+1} \subset_{\mu} B_{n}$. Summing up, we get

$$
C_{n+2} \subset D_{n} \subset_{\mu} E_{n} \subset_{\mu} A_{n} \subset C_{n+1} \subset P_{n+1} \subset_{\mu} B_{n} \subset_{\mu} Q_{n} \subset P_{n},
$$

and now it is clear that the sequences of bodies we have just constructed satisfy conditions (1)-(4) of Proposition 2.3. Finally, $B$ is the required body and satisfies condition (5). Indeed, notice that $K \subset(r / 2) \operatorname{int}(E) \subset r B$, and $P_{1} \subset(r / 2) E \subset 4 r B$.

CASE II: $X$ is reflexive. In this case it is known that there exists a continuous linear injection $T: X \rightarrow c_{0}(\Gamma)$ for some (infinite) set $\Gamma$ (see $[14$, p. 246], for instance). It is also well known that for an infinite set $\Gamma$, 
the space $c_{0}(\Gamma)$ is $c_{0}$-saturated, that is, every infinite-dimensional closed subspace of $c_{0}(\Gamma)$ has a closed subspace which is isomorphic to $c_{0}$. This clearly implies that $c_{0}(\Gamma)$ contains no closed infinite-dimensional reflexive subspaces. Therefore $Y:=\overline{T(X)} \subset c_{0}(\Gamma)$ is nonreflexive, and $T(X)$ is not a closed subspace of $Y \subset c_{0}(\Gamma)$. On the other hand, the space $c_{0}(\Gamma)$ has a $C^{\infty}$ smooth equivalent norm (see [14, Chapter V, Theorem 1.5]), whose restriction to $Y$ defines a $C^{\infty}$ smooth equivalent norm $|\cdot|$. Finally, it is well known [14] that the space $c_{0}(\Gamma)$ has $C^{\infty}$ smooth partitions of unity, hence so does $Y$.

Summing up, we have a continuous linear injection $T: X \rightarrow Y$, where $(Y,|\cdot|)$ is a nonreflexive Banach space with a $C^{\infty}$ smooth norm and $C^{\infty}$ smooth partitions of unity, and $T(X)$ is dense in $Y$.

Set $B^{\prime}=\{y \in Y:|y| \leq 1\}$, which is a $C^{\infty}$ smooth bounded convex body with $0 \in \operatorname{int}\left(B^{\prime}\right)$. Define $B=T^{-1}\left(B^{\prime}\right)$. It is clear that $B$ is a radially bounded $C^{\infty}$ smooth convex body.

Let $K$ be a weakly compact subset of $X$ and $r>0$ with $K \subset r B$. Since $T$ is continuous, $T(K)$ is weakly compact. Moreover $T(K) \subset T(r B) \subset r B^{\prime} \subset_{\mathrm{d}}$ $(r / 2)\left(4 B^{\prime}\right)$. Now we may copy the above proof (nonreflexive case), with $4 B^{\prime}=E$ and $T(K)$ replacing $K$, in order to obtain sequences of $C^{\infty}$ smooth starlike bodies, $\left(P_{n}^{\prime}\right),\left(C_{n}^{\prime}\right),\left(A_{n}^{\prime}\right),\left(B_{n}^{\prime}\right),\left(Q_{n}^{\prime}\right),\left(D_{n}^{\prime}\right),\left(E_{n}^{\prime}\right)$, and a sequence $\left(c_{n}^{\prime}\right)$ of points of $Y$ satisfying conditions (1)-(4) of the statement of Proposition 2.3 and $P_{1}^{\prime} \subset(r / 2)\left(4 B^{\prime}\right)=2 r B^{\prime}$. Ensure further that $c_{n}^{\prime} \in T(X) \cap \operatorname{int}\left(C_{n}^{\prime}\right)$ for each $n \in \mathbb{N}$ (this is possible because $T(X)$ is dense in $Y$, hence $T(X) \cap$ $\operatorname{int}\left(C_{n}^{\prime}\right) \neq \emptyset$ for all $\left.n\right)$.

Then, for each $n \in \mathbb{N}$, define $c_{n}=T^{-1}\left(c_{n}^{\prime}\right) \in X$, and

$$
\begin{array}{lll}
C_{n}=T^{-1}\left(C_{n}^{\prime}\right), & B_{n}=T^{-1}\left(B_{n}^{\prime}\right), & P_{n}=T^{-1}\left(P_{n}^{\prime}\right), \quad A_{n}=T^{-1}\left(A_{n}^{\prime}\right), \\
Q_{n}=T^{-1}\left(Q_{n}^{\prime}\right), & D_{n}=T^{-1}\left(D_{n}^{\prime}\right), & E_{n}=T^{-1}\left(E_{n}^{\prime}\right) \subset X .
\end{array}
$$

By Lemma 2.8, these are radially bounded $C^{\infty}$ smooth starlike bodies with respect to $c_{n+2}$. On the other hand, Lemma 2.9 guarantees that

$$
C_{n+2} \subset D_{n} \subset_{\mu} E_{n} \subset_{\mu} A_{n} \subset C_{n+1} \subset P_{n+1} \subset B_{n} \subset_{\mu} Q_{n} \subset P_{n} .
$$

Finally, it is immediately checked that $\bigcap_{n=1}^{\infty} C_{n}=\emptyset, \bigcap_{n=1}^{\infty} P_{n}=K, P_{1}=$ $T^{-1}\left(P_{1}^{\prime}\right) \subset T^{-1}\left(2 r B^{\prime}\right)=2 r B \subset 4 r B$.

Now we are in a position to finish the proof of the main result.

Proof of Theorem 1.1. We may assume that $A$ is a bounded starlike body with respect to the origin. Let $B$ be the radially bounded $C^{p}$ smooth starlike body provided by Proposition 2.3. Choose $r>0$ such that $A \subset_{\mu} r B$. Bearing in mind that $K \subset_{\mathrm{d}} A \subset_{\mu} r B$, it follows from Proposition 2.3 that there are sequences $\left(P_{n}\right),\left(C_{n}\right),\left(A_{n}\right),\left(B_{n}\right),\left(Q_{n}\right),\left(D_{n}\right),\left(E_{n}\right)$ of subsets of $X$ and a sequence $\left(c_{n}\right)$ of points of $X$ which satisfy the conditions of Proposition 2.1. 
Then we can apply this proposition to find a $C^{p}$ diffeomorphism $\Psi: X \rightarrow$ $X \backslash K$ such that $\Psi$ is the identity on $X \backslash P_{1} \supset X \backslash 4 r B$.

On the other hand, since $K \subset_{\mathrm{d}} A$, Lemma 2.11 allows us to find two $C^{p}$ smooth starlike bodies $U_{1}, U_{2}$ with respect to 0 such that $K \subset U_{1} \subset_{\mu}$ $U_{2} \subset A$. Now, by the Four Bodies Lemma 2.2, there is a $C^{p}$ diffeomorphism $g: X \rightarrow X$ such that $g\left(U_{2}\right)=4 r B$ and $g$ is the identity on $U_{1} \supset K$; notice in particular that $g(K)=K$.

Define then $h=g^{-1} \circ \Psi \circ g$. It is clear that $h$ is a $C^{p}$ diffeomorphism from $X$ onto $X \backslash K$. Moreover, if $x \in X \backslash A$ then $x \notin U_{2}$, so $g(x) \notin 4 r B$, which implies that $\Psi(g(x))=g(x)$, hence $h(x)=x$; that is, $h$ is the identity off $A$.

Acknowledgements. The authors thank Tadeusz Dobrowolski for several helpful suggestions which improved the exposition of these results.

\section{References}

[1] D. Azagra, Diffeomorphisms between spheres and hyperplanes in infinite-dimensional Banach spaces, Studia Math. 125 (1997), 179-186.

[2] D. Azagra and M. Cepedello, Uniform approximation of continuous mappings by smooth mappings with no critical points on Hilbert manifolds, Duke Math. J., to appear.

[3] D. Azagra and R. Deville, James' theorem fails for starlike bodies, J. Funct. Anal. 180 (2001), 328-346.

[4] D. Azagra and T. Dobrowolski, Smooth negligibility of compact sets in infinitedimensional Banach spaces, with applications, Math. Ann. 312 (1998), 445-463.

[5] - - - Real-analytic negligibility of points and subspaces in infinite-dimensional Banach spaces, with applications, Canad. Math. Bull. 45 (2002), 3-11.

[6] - - - On the topological classification of starlike bodies in Banach spaces, Topology Appl. 132 (2003), 221-234.

[7] D. Azagra and M. Jiménez-Sevilla, The failure of Rolle's Theorem in infinite dimensional Banach spaces, J. Funct. Anal. 182 (2001), 207-226.

[8] C. Bessaga, Every infinite-dimensional Hilbert space is diffeomorphic with its unit sphere, Bull. Acad. Polon. Sci. Sér. Sci. Math. Astronom. Phys. 14 (1966), 27-31.

[9] -, Interplay between infinite-dimensional topology and functional analysis. Mappings defined by explicit formulas and their applications, Topology Proc. 19 (1994), $15-35$.

[10] C. Bessaga and V. L. Klee, Two topological properties of topological linear spaces, Israel J. Math. 2 (1964), 211-220.

[11] - - - Every non-normable Fréchet space is homeomorphic with all of its closed convex bodies, Math. Ann. 163 (1966), 161-166.

[12] C. Bessaga and A. Pełczyński, Selected Topics in Infinite-Dimensional Topology, Monografie Mat. 58, PWN, Warszawa, 1975.

[13] D. Burghelea and N. H. Kuiper, Hilbert manifolds, Ann. of Math. 90 (1969), 379-417.

[14] R. Deville, G. Godefroy, and V. Zizler, Smoothness and Renormings in Banach Spaces, Pitman Monogr. Surveys Pure Appl. Math. 64, Longman, Harlow, 1993. 
[15] T. Dobrowolski, Smooth and $\mathbb{R}$-analytic negligibility of subsets and extension of homeomorphisms in Banach spaces, Studia Math. 65 (1979), 115-139.

[16] - Every infinite-dimensional Hilbert space is real-analytically isomorphic with its unit sphere, J. Funct. Anal. 134 (1995), 350-362.

[17] J. Eells and K. D. Elworthy, Open embeddings of certain Banach manifolds, Ann. of Math. 91 (1970), 465-485.

[18] R. Haydon, A counterexample to several questions about scattered compact spaces, Bull. London Math. Soc. 22 (1990), 261-268.

[19] —, Trees in renorming theory, Proc. London Math. Soc. (3) 78 (1999), 541-584.

[20] V. L. Klee, Convex bodies and periodic homeomorphisms in Hilbert space, Trans. Amer. Math. Soc. 74 (1953), 10-43.

[21] - Some topological properties of convex sets, Trans. Amer. Math. Soc. 78 (1955), $30-45$.

[22] N. Moulis, Sur les variétés hibertiennes et les fonctions non-dégénérées, Indag. Math. 30 (1968), 497-511.

[23] C. A. Riley, Negligibility in nonlocally convex spaces, Proc. Amer. Math. Soc. 41 (1973), 619-624.

Departamento de Análisis Matemático

Facultad de Ciencias Matemáticas

Universidad Complutense

28040 Madrid, Spain

E-mail: daniel_azagra@mat.ucm.es a_montesinos@mat.ucm.es

Received September 3, 2002

Revised version January 12, 2004 